\title{
Three-dimensional stability of an elliptical vortex in a straining field
}

\author{
By A. C. ROBINSON†AND P. G. SAFFMAN \\ Applied Mathematics, California Institute of Technology, Pasadena, California 91125 \\ (Received 8 September 1983 and in revised form 30 January 1984)
}

The three-dimensional linear stability of a rectilinear vortex of elliptical cross-section existing as a steady state in an irrotational straining field is studied numerically in the case of finite strain. It is shown that the instability predicted analytically for weak strain persists for finite strain and that the weak-strain results continue to be quantitatively valid for finite strain. The dependence of the growth rates of the unstable modes on the strain and the axial-disturbance wavelength is discussed. It is also shown that a three-dimensional instability is always more unstable than a two-dimensional instability in the range of parameters of most interest.

\section{Introduction}

Moore \& Saffman (1971) demonstrated that in the theory of inviscid incompressible flow a steady rectilinear uniform vortex of elliptical cross-section could exist in a uniform straining field in unbounded fluid. We wish to study the three-dimensional linear instability of the Moore-Saffman vortex and discuss the dependence of several unstable modes on the strain and the axial wavelength. Our results are obtained numerically and extend previous asymptotic and perturbation analyses to the case of finite strain.

Our results are intended to shed further light on the fundamental mechanisms for the three-dimensional instability of general vortex arrays. The computations of Pierrehumbert (1980) and Pierrehumbert \& Widnall (1982) on the three-dimensional stability of a single straight row and doubly periodic infinite arrays of vortices with both top-hat and continuously distributed vorticity are important pioneering studies. In a previous paper (Robinson \& Saffman 1982), we discussed the three-dimensional instabilities of the single row, the staggered double row and the symmetric double row of vortices using the Biot-Savart formulation with a suitable cutoff to compute the self-induced motion of each vortex. This is an asymptotic formulation which is restricted to long-axial-wavelength disturbances and well-separated vortices and gives unstable modes relating to the mutual interactions of the vortices. In the present paper we shall discuss the effect of strain and finite area on the Biot-Savart long-axial-wavelength unstable mode. Furthermore, mutual interaction effects are small for axial-disturbance wavelengths on the order of the vortex core, and the primary mechanism for instability is the interaction of the vortex core with the steady-state straining field of the other vortices in the array. For this reason we discuss the effect of finite strain on the short-axial-wavelength instability first predicted for weak strain by the arguments of Widnall, Bliss \& Tsai (1974) and demonstrated qualitatively and quantitatively by the perturbation analyses of Moore

$\dagger$ Present address: Sandia National Laboratories, Albuquerque, New Mexico 87185. 


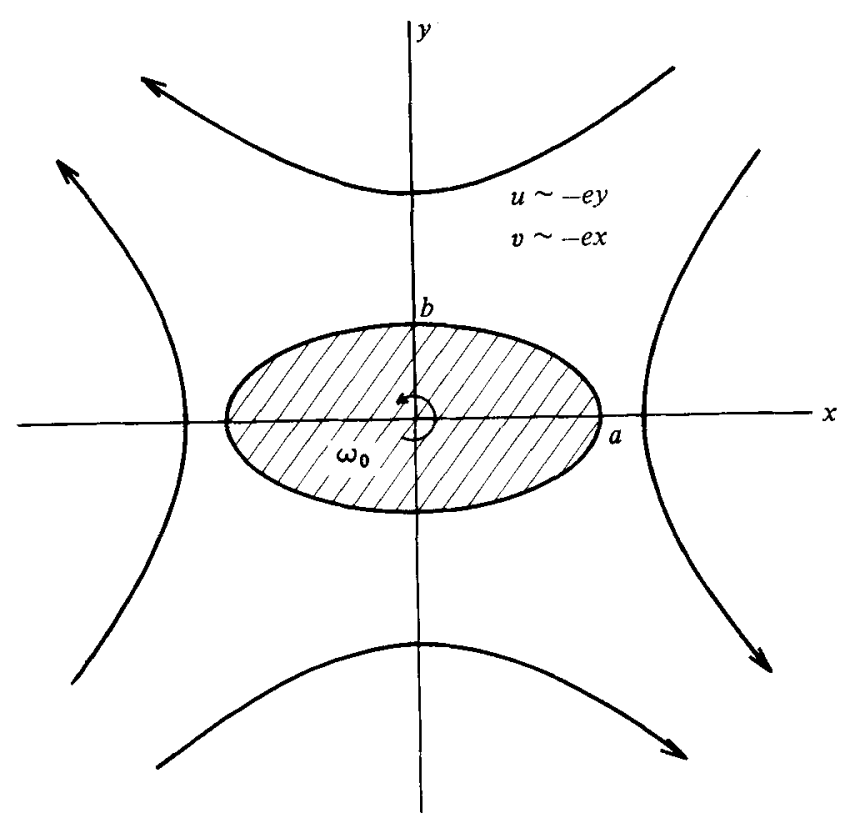

Figure 1. Cross-section of steady uniform elliptical vortex in an irrotational straining field.

\& Saffman (1975) and Tsai \& Widnall (1976). The weak strain analysis has proved successful in explaining the observed instability of vortex rings (Widnall \& Tsai 1977; Saffman 1978).

We outline in $\$ \S 2$ and 3 the properties of the exact steady solution and the known linearized stability results for special cases. In $\$ 4$ and 5 we discuss the formulation of the linear stability equations and corresponding regularity and boundary conditions. The numerical method used to solve the stability equations for the normal modes and growth rates is described in $\S 6$. In $\$ 7$ we discuss the results of the computations.

\section{The steady state}

Moore \& Saffman (1971) obtained a steady solution of the equations of motion for an inviscid incompressible flow in which a rectilinear vortex with uniform vorticity $\omega_{0}$, aligned along the axis, exists in a uniform irrotational straining field of infinite extent. Unsteady two-dimensional solutions have been given by Kida (1981) for elliptical deformations. His solutions have since been discussed further and extended to the case of a three-dimensional irrotational straining field (Neu 1983). However, our interest lies in the three-dimensional instabilities of the steady vortex. The steady solution is shown schematically in cross-section in figure 1 . The solution for the stream function in the interior of the ellipse is

$$
\Psi=-\frac{1}{2} \Omega a b\left(\frac{x^{2}}{a^{2}}+\frac{y^{2}}{b^{2}}-1\right), \quad \Omega=\frac{\omega_{0} a b}{a^{2}+b^{2}},
$$

where $a$ and $b$ are respectively the semimajor and semiminor axes of the ellipse. Matching to the irrotational outer flow, as described in detail by Moore \& Saffman, gives a requirement on the shape of the ellipse. If $\theta=a / b$ then

$$
\frac{e}{\omega_{0}}=\frac{\theta(\theta-1)}{\left(\theta^{2}+1\right)(\theta+1)}
$$


The strain as a function of $\theta$ has a single maximum, $e / \omega_{0} \approx 0.15$, at $\theta_{\mathrm{cr}} \approx 2.9$. For $e / \omega_{0} \lesssim 0.15$ there are two possible steady solutions, one more elongated than the other, and none for $e / \omega_{0}$ greater than this value.

It is convenient to express the interior flow field in elliptic-cylinder coordinates defined by

$$
\left.\begin{array}{rl}
x & =c \cosh \xi \cos \eta \quad(0 \leqslant \xi \leqslant \infty), \\
y & =c \sinh \xi \sin \eta \quad(-\pi \leqslant \eta \leqslant \pi), \\
h^{2} & =\frac{1}{2} c^{2}(\cosh 2 \xi-\cos 2 \eta),
\end{array}\right\}
$$

where $c=\left(a^{2}-b^{2}\right)^{\frac{1}{2}}$ is the semifocal length. The line element $h$ is the same for both the $\xi$-and $\eta$-coordinates. The steady interior flow is

$$
\begin{aligned}
& \left.h U_{\xi}=\frac{\partial \Psi}{\partial \eta}=\frac{1}{4} \omega_{0} c^{2}(1-f(\theta) \cosh 2 \xi)\right) \sin 2 \eta, \\
& h U_{\eta}=-\frac{\partial \Psi}{\partial \xi}=\frac{1}{4} \omega_{0} c^{2}(1-f(\theta) \cos 2 \eta) \sinh 2 \xi
\end{aligned}
$$

where $f(\theta)=\left(\theta^{2}-1\right) /\left(\theta^{2}+1\right)$, and $U_{\xi}$ and $U_{\eta}$ are velocities in the $\xi$ - and $\eta$-directions respectively. The above solution if valid for $0 \leqslant \xi \leqslant \xi_{0}$, where $\xi_{0}$ defines the boundary of the ellipse. Note that $h U_{\xi}$ and $h U_{\eta}$ are smooth functions of the coordinates $\xi$ and $\eta$. This is true in general. That is, suppose in Cartesian coordinates we have a vector function

$$
\boldsymbol{U}=U_{x}(x, y, z) \boldsymbol{i}+U_{y}(x, y, z) \boldsymbol{j}+U_{z}(x, y, z) \boldsymbol{k}
$$

where $U_{x}, U_{y}$ and $U_{z}$ are all smooth functions of $x, y$ and $z$ with convergent Taylor series. If we write this vector in terms of elliptic-eylinder coordinates then

$$
\begin{aligned}
U=\left(U_{x} \sinh \xi \cos \eta+U_{y} \cosh \xi \sin \eta\right) \frac{c}{h} \xi \\
+\left(U_{y} \sinh \xi \cos \eta-U_{x} \cosh \xi \sin \eta\right) \frac{c}{h} \hat{\eta}+U_{z} \hat{z}
\end{aligned}
$$

Thus $h U_{\xi}$ and $h U_{\eta}$ are smooth functions of $\xi$ and $\eta$. It should perhaps be emphasized that the velocities are not related to functions of the complex variable $\xi+i \eta$ as in inviscid irrotational-flow theory.

\section{Linearized stability - limiting cases}

We describe briefly here the known results for the stability of the Moore-Saffman vortex to two- and three-dimensional disturbances. These results are limited to the boundaries of the parameter space which we wish to study. In their original paper Moore \& Saffman computed the stability of the vortex to two-dimensional disturbances characterized by a mode number $m>0$ giving the angular dependence in ellipticcylinder coordinates. In this case the growth rate $\sigma$ is given by the formula

$$
\frac{\sigma^{2}}{\omega_{0}^{2}}=-\frac{1}{4}\left\{\left(\frac{2 m \theta}{\theta^{2}+1}-1\right)^{2}-\left(\frac{\theta-1}{\theta+1}\right)^{2 m}\right\},
$$

and in the case $m=1$ we have

$$
\frac{\sigma^{2}}{\omega_{0}^{2}}=\frac{\theta^{2}(\theta-1)^{2}}{(\theta+1)^{2}\left(\theta^{2}+1\right)^{2}}=\frac{e^{2}}{\omega_{0}^{2}}>0 .
$$




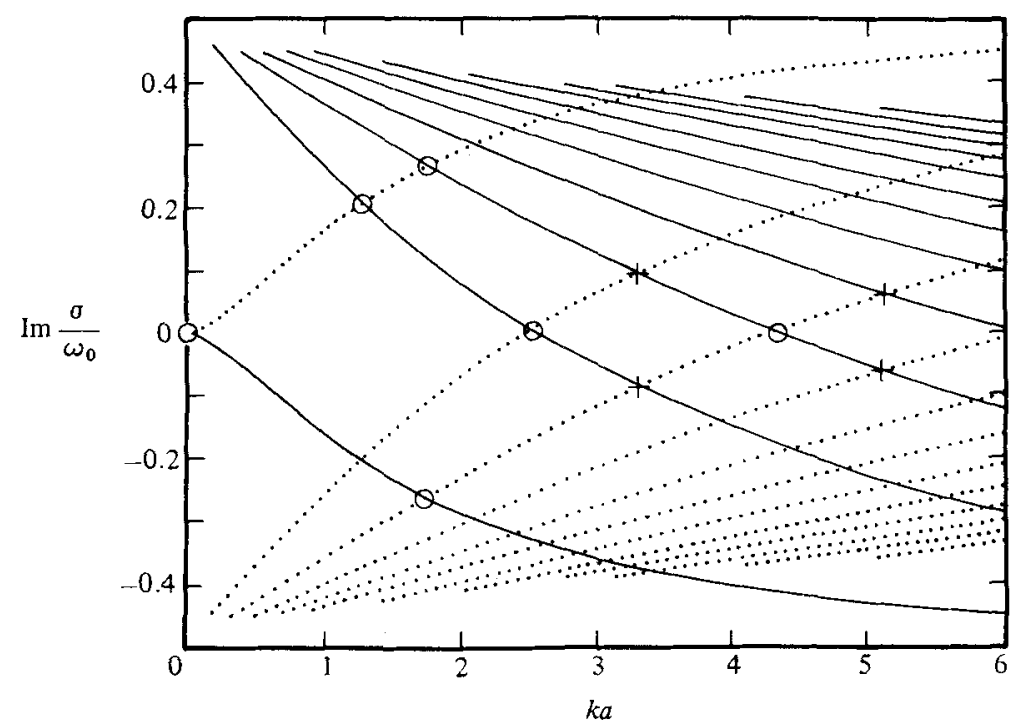

Figure 2. Roots of the dispersion relation for angular mode number $m=+1$ (dotted line) and $m=-1$ (solid line) for uniform circular vortex. Circles indicate unstable crossing points and crosses stable crossing points in weak strain. (After Tsai \& Widnall (1976).)

Thus the $m=1$ mode is always unstable and corresponds to a translation of the ellipse outward along one of the principal axes of strain. For $m>1$ and $\theta<\theta_{\mathbf{c r}}, \sigma^{2}<0$ and the vortex is thus structurally stable to two-dimensional disturbances. Moore \& Saffman also computed the effect of long-axial-wavelength three-dimensional disturbances on the $m=1$ mode and concluded that the effect is to reduce the growth rate of the instability.

The $\theta \equiv 1$ case was discussed many years ago by Kelvin (1880). For disturbances proportional to $\exp (\sigma t+\mathrm{i} m \phi+\mathrm{i} k z)$ the dispersion relation giving $\sigma(k, m)$ for waves on a rectilinear uniform circular vortex is

$$
\frac{J_{|m|}^{\prime}(g)}{g J_{|m|}(g)}+\frac{m}{g^{2} \gamma}=-\frac{K_{|m|}^{\prime}(k a)}{k a K_{|m|}(k a)}
$$

where $m$ is the angular mode number in cylindrical polar coordinates, $k$ is the axial wavenumber, $a$ is the radius of the vortex, and $g^{2}=(k a)^{2}\left(1-\gamma^{2}\right) / \gamma^{2}$, with $\gamma=\frac{1}{2} m-\mathrm{i} \sigma / \omega_{0}$. The function $J$ is the Bessel function of the first kind and $K$ is the modified Bessel function of the second kind. According to Kelvin, roots of (3.3) are pure imaginary and give the frequency of stable oscillations of the unstrained vortex. The roots arise from solutions with $\gamma$ in $(-1,1)$ so that $-\mathrm{i} \sigma / \omega_{0}=\operatorname{Im}\left(\sigma / \omega_{0}\right)$ lies in $\left(-1-\frac{1}{2} m, 1-\frac{1}{2} m\right)$. There are an infinite number of roots for each $m$ and $k$. Figure 2 shows a plot of some of the $|m|=1$ roots as a function of $k a$.

Moore \& Saffman (1975) pointed out the possibility of parametric instability in a weak straining field for values of $k$ near the crossing points shown in figure 2 , and Tsai \& Widnall (1976) calculated the effect for a uniform vortex. They found that at some of the crossing points the eigenvalues would become unstable and gave quantitative values for the change in the eigenvalues and the width in $k$ of the region of instability. The results of Tsai \& Widnall allow precise checks to be made on the numerical method used to calculate the modes of instability for finite values of the strain. 


\section{Linearized stability equations}

The three-dimensional stability of the finitely strained Moore-Saffman vortex will be studied by finding normal modes of the linearized equations. As the flow inside the vortex is rotational, the resulting normal modes will be rotational and the interior flow must be described by the Euler equations. Outside the vortex the flow is irrotational, and owing to Kelvin's theorem the perturbed motion will be irrotational also. We can therefore describe the exterior flow by a velocity potential satisfying Laplace's equation. We have also boundary conditions matching the interior to the exterior flow. Since the surface bounding the rotational fluid is material, we obtain the usual kinematic condition specifying normal velocity. Continuity of tangential velocity on the boundary of the vortex insures continuity of the pressure. The linearized boundary conditions are derived by expansion about the undisturbed boundary of the exact nonlinear boundary condition satisfied on the disturbed boundary. The choice of coordinate system is thus crucial to the simplicity of the analytical statement of the linearized boundary condition as well as its numerical implementation. Since the boundary of the vortex is an ellipse, it is natural to use elliptic-cylinder coordinates in both the exterior and interior of the ellipse as this makes implementation of the boundary conditions on the surface of the vortex extremely simple. There is a disadvantage in that a coordinate singularity is introduced at $|x|=c$ in the interior of the ellipse. However, this poses little difficulty, as will be shown in $\S 5$.

The Eulerian equations of motion in elliptic-cylinder coordinates can be derived from standard relations for orthogonal curvilinear coordinates (Batchelor 1967). We define $u_{\xi}, u_{\eta}, \bar{u}_{z}$ and $\bar{p}$ to be respectively the velocities in the subscripted coordinate directions and the pressure. However, it is convenient for numerical purposes to rewrite the equations so that the dependent variables are smooth functions of $\xi$ and $\eta$. To this end we set $\bar{u}_{1}=h u_{\xi}$ and $\bar{u}_{2}=h u_{\eta}$ and, for convenience of notation, $x_{1}=\xi$ and $x_{2}=\eta$. This allows the use of summation convention for the indices 1 and 2 . The resulting equations are

$$
\begin{gathered}
\frac{1}{h^{2}} \frac{\partial \bar{u}_{j}}{\partial x_{j}}+\frac{\partial \bar{u}_{z}}{\partial z}=0 \\
\frac{\partial \bar{u}_{i}}{\partial t}+\frac{\bar{u}_{j}}{h^{2}} \frac{\partial \bar{u}_{i}}{\partial x_{j}}+\bar{u}_{z} \frac{\partial \bar{u}_{i}}{\partial z}+\frac{\bar{u}_{j}^{2}}{2} \frac{\partial}{\partial x_{i}}\left(\frac{1}{h^{2}}\right)=-\frac{\partial \bar{p}}{\partial x_{i}}, \quad i=1,2, \\
\frac{\partial \bar{u}_{z}}{\partial t}+\frac{\bar{u}_{j}}{h^{2}} \frac{\partial \bar{u}_{z}}{\partial x_{j}}+\bar{u}_{z} \frac{\partial \bar{u}_{z}}{\partial z}=-\frac{\partial \bar{p}}{\partial z} .
\end{gathered}
$$

We now look for normal modes of the linearized equations by taking perturbations of the following form,

$$
\left.\begin{array}{rl}
\bar{u}_{n} & =U_{n}\left(x_{1}, x_{2}\right)+u_{n}\left(x_{1}, x_{2}\right) \mathrm{e}^{\sigma t+\mathrm{i} k z}, \quad n=1,2, \\
\bar{u}_{z} & =u_{z}\left(x_{1}, x_{2}\right) \mathrm{e}^{\sigma t+\mathrm{i} k z}, \\
\bar{p} & =P\left(x_{1}, x_{2}\right)+p\left(x_{1}, x_{2}\right) \mathrm{e}^{\sigma t+\mathrm{i} k z} .
\end{array}\right\}
$$

The steady-state velocities (2.4), (2.5) and pressure are represented by capitalized quantities. Inserting (4.4) into the equations and dropping all terms of second order in the perturbations, we obtain a set of linear equations for the perturbations $u_{1}, u_{2}$, $u_{z}$ and $p$. Solving for $u_{z}$ in the continuity equation (4.1), we can eliminate $u_{z}$ explicitly by substitution into the third equation of motion (4.3). This gives an expression for 
$p$, which can then be eliminated through the first two equations of motion (4.2). The resulting system of coupled linear equations for $u_{1}$ and $u_{2}$ can be expressed as follows:

$$
\left\{A_{i j}-k^{2}\left(C_{i j}+D_{i j}\right)\right\} u_{j}=\sigma\left\{k^{2} \delta_{i j}-B_{i j}\right\} u_{j}, \quad i=1,2,
$$

where

$$
\begin{gathered}
A_{i j}(\cdot)=\frac{\partial}{\partial x_{i}}\left\{\frac{U_{m}}{h^{2}} \frac{\partial}{\partial x_{m}}\left(\frac{1}{h^{2}} \frac{\partial(\cdot)}{\partial x_{j}}\right)\right\}, \quad B_{i j}\left(\bullet^{\cdot}\right)=\frac{\partial}{\partial x_{i}}\left(\frac{1}{h^{2}} \frac{\partial(\cdot)}{\partial x_{j}}\right), \\
C_{i j}\left(\bullet^{\cdot}\right)=\left\{\frac{\partial}{\partial x_{i}}\left(\frac{1}{h^{2}} U_{j}\right)+(i-j) \omega_{0}\right\}(\cdot), \quad D_{i j}(\cdot)=\delta_{i j} \frac{U_{m}}{h^{2}} \frac{\partial(\cdot)}{\partial m} .
\end{gathered}
$$

The above constitute the linearized disturbance equations for the interior flow.

The exterior irrotational flow is described by a velocity potential $\bar{\phi}$ satisfying Laplace's equation. Proceeding as before we let $\bar{\phi}=\Phi\left(x_{1}, x_{2}\right)+\phi\left(x_{1}, x_{2}\right) \mathrm{e}^{\sigma t+\mathrm{i} k z}$. The perturbation $\phi$ satisfies

$$
\frac{1}{h^{2}}\left(\frac{\partial^{2} \phi}{\partial x_{n}^{2}}\right)-k^{2} \phi=0
$$

and the solutions of this equation via the method of separation of variables are products of Mathieu functions.

\section{Regularity and boundary conditions}

We describe first the regularity conditions to be satisfied by the normal modes at $\xi=0$ due to the singularity in the coordinate system. It is clear that all dependent variables must be $2 \pi$-periodic in $x_{2}=\eta$, the angular coordinate. In the radial coordinate $x_{1}=\xi$ the situation is more complicated. At $\xi=0$ we must require that the velocity be smooth in a non-singular coordinate system. By inserting values for $x$ and $y$ given by (2.3) into (2.7) and examining the numerators in (2.7), it is clear (allowing $\xi<0$ for the moment) that the barred velocities must satisfy

$$
\left.\begin{array}{rl}
\bar{u}_{m}(\xi, \eta) & =-\bar{u}_{m}(-\xi,-\eta), \quad m=1,2, \\
\bar{u}_{z}(\xi, \eta) & =\bar{u}_{z}(-\xi,-\eta)
\end{array}\right\}
$$

This implies the following relations at $\xi=0$ :

$$
\begin{aligned}
& \frac{\partial^{n} \bar{u}_{m}}{\partial \xi^{n}}(0, \eta)=(-1)^{n+1} \frac{\partial^{n} \bar{u}_{m}}{\partial \xi^{n}}(0,-\eta), \quad m=1,2, \\
& \frac{\partial^{n} \bar{u}_{z}}{\partial \xi^{n}}(0, \eta)=(-1)^{n} \frac{\partial^{n} \bar{u}_{z}}{\partial \xi^{n}}(0,-\eta)
\end{aligned}
$$

for all non-negative integers $n$. Since the steady flow satisfies these relations and the relations are linear, the perturbations must also satisfy them.

The linearized boundary conditions at the surface of the vortex require some care. We parametrize the surface of the vortex by

$$
\xi=\xi_{0}+F(\eta) \mathrm{e}^{\sigma t+\mathrm{i} k z}
$$

where $F$ is the small quantity. The kinematic condition giving the motion of the boundary of the vortex is then

$$
\frac{\mathrm{D}}{\mathrm{D} t}\left(\xi-\xi_{0}-F(\eta) \mathrm{e}^{\sigma t+\mathrm{i} k z}\right)=0 \quad \text { at } \quad \xi=\xi_{0}+F(\eta) \mathrm{e}^{\sigma t+\mathrm{i} k z}
$$

The dynamic boundary condition requires continuity of tangential velocity on the disturbed boundary. Substitution of the assumed form of the velocities and expanding 
in small quantities about $\xi_{0}$ gives the following conditions at $\xi_{0}$ (see Moore \& Saffman 1971):

$$
\begin{gathered}
\frac{\partial \phi}{\partial \xi}=\sigma h^{2} F+\Omega \frac{\partial}{\partial \eta}\left(h^{2} F\right), \\
u_{1}=\sigma h^{2} F+\Omega \frac{\partial}{\partial \eta}\left(h^{2} F\right), \\
\frac{\partial \phi}{\partial \eta}-u_{2}=\omega_{0} h^{2} F \\
\frac{\partial^{2} \phi}{\partial \xi^{2}}-\frac{\partial u_{1}}{\partial \xi}=-\omega_{0} \frac{\partial}{\partial \eta}\left(h^{2} F\right) .
\end{gathered}
$$

The first three conditions are exactly the equations derived by Moore \& Saffman for the two-dimensional stability problem. By use of the continuity equation, the last condition (5.8) can be seen to be equivalent to continuity of axial perturbation velocity. Since we have assumed no steady axial flow in the vortex, it should be clear that the addition of $(5.8)$ to $(5.5)-(5.7)$ gives the correct set of linearized boundary conditions for the full three-dimensional stability problem as no coupling of steadystate and perturbation quantities occurs in the axial direction at first order.

In the exterior region we require the perturbation velocity to decay exponentially as $x^{2}+y^{2} \rightarrow \infty$. The appropriate solutions to the reduced equation (4.6) are products of Mathieu functions, $K e_{n}(\xi ; q) c e_{n}(\eta ;-q)$ and $K o_{n}(\xi ; q) s e_{n}(\eta ;-q)$, where we have used the notation of Abramowitz \& Stegun (1972) and the parameter $q=\frac{1}{4}(c k)^{2}$.

\section{Numerical method}

The computation of the eigenvalues is accomplished by means of a straightforward collocation scheme similar to the kind recommended by Boyd (1978) for eigenvalue problems of our type and used with success for example by Pierrehumbert \& Widnall (1982) in their calculations.

An examination of the coefficients in the linearized equations (4.5) and (4.6) shows that the only $\eta$-dependence is through either $\cos 2 \eta$ or $\sin 2 \eta$. This indicates that the normal modes must separate into $\pi$-and $2 \pi$-periodic solutions just as in the standard analysis of the periodic solutions of Mathieu's equation. We will study only $2 \pi$-periodic unstable modes, and, of these, specifically the ones that correspond to the zero-crossings of the eigenvalues in the case of the circular vortex. These angular modes have azimuthal dependence $|m|=1$ in (3.3). There are several reasons to limit the computations to this restricted subcase. As noted in $\S 3$, there exists an infinite number of pure imaginary roots for each $m$ and $k$ in the unstrained case. For the $2 \pi$-periodic case we may have for special values of $k$ a pair of physically distinguishable modes with the same frequency provided that the angular mode numbers satisfy $\left(m_{1}, m_{2}\right)=(1,-1),(1,3),(-1,-3),(3,5)$, ete. This is seen from the discussion of the location of the roots given in $\S 3$. For the $\pi$-periodic case we have similarly $\left(m_{1}, m_{2}\right)=(1,2),(0,-2),(2,4),(-2,-4)$, etc. There is an infinite number of such crossing points for each pair $\left(m_{1}, m_{2}\right)$. Moore \& Saffman (1975) give a good discussion of why such crossing points may become unstable at small strain and they showed that, under assumptions satisfied by the Moore-Saffman vortex, the zero-crossing eigenvalues of the $|m|=1$ modes would become unstable. For eigenvalues which cross at non-zero values the effect of strain may or may not be destabilizing. Tsai \& Widnall (1976) gave specific results for the $\left(m_{1}, m_{2}\right)=(+1,-1)$ case as in figure 2 . They found, 
however, that the growth rates of the instabilities for the eigenvalues at nonzero-crossings were an order of magnitude smaller than the zero-crossing instabilities. In the $\left(m_{1}, m_{2}\right)=(0,2),(0,-2)$ cases the eigenvalues do not cross at zero frequency but do cross at non-zero frequencies, so that these may also become unstable. Practical considerations dictate that some decision be made on the eigenmodes and eigenvalues to be calculated. We expect only those modes with the least structure in both the azimuthal and radial directions and with the largest growth rates to be of physical relevance since presumably viscous effects come into play at the smaller scales. We thus make the plausible assumption that at finite strain the most important unstable modes continue to be the ones arising from the zero-crossings of the $|m|=1$ modes at infinitesimal strain and that the instabilities which may arise from other resonances in both the $2 \pi$ - and $\pi$-periodic cases at non-zero-crossing points of the eigenvalues are of less importance.

Recalling the conditions (5.2) on the interior solutions, we now represent the $2 \pi$-periodic modes in the following form:

$$
\left.\begin{array}{rl}
u_{i} & =c_{m n}^{i} T_{2 n+1}\left(\frac{\xi}{\xi_{0}}\right) \cos (2 m+1) \eta+d_{m n}^{i} T_{2 n}\left(\frac{\xi}{\xi_{0}}\right) \sin (2 m+1) \eta, \quad i=1,2, \\
\phi & =c_{m}^{3} \frac{K e_{2 m+1}(\xi, q)}{K e_{2 m+1}\left(\xi_{0}, q\right)} c e_{2 m+1}(\eta,-q)+d_{m}^{3} \frac{K o_{2 m+1}(\xi, q)}{K o_{2 m+1}\left(\xi_{0}, q\right)} s e_{2 m+1}(\eta,-q), \\
h^{2} F & =c_{m}^{4} \cos (2 m+1) \eta+d_{m}^{4} \sin (2 m+1) \eta,
\end{array}\right\}
$$

with the summation convention used for $m=0,1, \ldots, N_{\mathrm{A}}-1$ and $n=0,1, \ldots, N_{\mathrm{R}}-1$. $T_{m}$ are standard Tchebyscheff polynomials. With this representation we now require that the interior equations $(4.5)$ be satisfied at the points $\left(\xi_{i}, \eta_{j}\right)$, where

$$
\left.\begin{array}{l}
\frac{\xi_{i}}{\xi_{0}}=\cos \frac{\pi(2 i-1)}{4\left(N_{\mathrm{R}}-1\right)}, \quad i=1,2, \ldots, N_{\mathrm{R}}-1, \\
\eta_{j}=\frac{\pi(j-1)}{2 N_{\mathrm{A}}}, \quad j=1,2, \ldots, 2 N_{\mathrm{A}} .
\end{array}\right\}
$$

To accomplish this, the equations represented in compact form by (4.5) are fully expanded and the explicit singularities in the equations removed by multiplying through by $h^{8}$. We also require that the boundary conditions (5.5)-(5.8) be satisfied at the points $\eta_{j}$.

The above collocation scheme leads to a generalized eigenvalue problem for the growth rates $\sigma$ and eigenvectors $\boldsymbol{x}=\left(c_{m n}^{1}, d_{m n}^{1}, c_{m n}^{2}, d_{m n}^{2}, c_{m}^{3}, d_{m}^{3}, c_{m}^{4}, d_{m}^{4}\right)^{\mathrm{T}}$ of the form

$$
\boldsymbol{A x}=\sigma \boldsymbol{B} \boldsymbol{x}
$$

with the matrices $\boldsymbol{A}$ and $\boldsymbol{B}$ coming from the collocation equations. The size of the system is $N=4 N_{\mathrm{A}}\left(N_{\mathrm{R}}+1\right)$. Eigenvalues of interest were computed initially using the EISPACK QZ-algorithm (Garbow et al. 1977) and the grading portion of the preprocessor described by Ward (1981). Since the desired eigenvalues are small, it was convenient to solve for $1 / \sigma$ rather than $\sigma$. The $\mathrm{QZ}$-algorithm tends to produce the largest eigenvalues first, so that in this way it was a simple matter to isolate the appropriate eigenvalues and eigenvectors. Once a single eigenvalue and eigenvector of interest were found then the parametric dependence on $k$ and $\theta$ was determined by extending the system through the normalization condition $x^{\mathrm{T}} \boldsymbol{x}-1=0$ and solving for both the eigenvalue and eigenvector via a Newton-chord method. The accuracy of the computations was checked by increasing the number of modes in the 
truncated expansions and by comparison with limiting results. A useful rule of thumb which became apparent was that the accuracy of a given eigenvalue was on the order of the size of the coefficients of the higher-order modes in the $u_{i}$ expansions.

Some comment should be made concerning the computation of the Mathieu functions. The characteristic values as well as the functional values of the angular Mathieu functions were computed from their Fourier-series representation using appropriate routines and/or modifications of the routines given by Clemm (1969); see also Sale (1970). These routines are based on algorithms given by Blanch (1966). The radial Mathieu function can be calculated once the corresponding characteristic values for the periodic solutions are known. The equation for the radial Mathieu function is

$$
\frac{\mathrm{d}^{2} y}{\mathrm{~d} \xi^{2}}-(\alpha+2 q \cosh 2 \xi) y=0
$$

where $\alpha$ is the characteristic value from the angular equation. Making the change of variable $z=q^{\frac{1}{2}} \mathrm{e}^{\xi}$, the equation becomes

$$
\frac{\mathrm{d}^{2} y}{\mathrm{~d} z^{2}}+\frac{1}{z} \frac{\mathrm{d} y}{\mathrm{~d} z}-\left[1+\frac{\alpha}{z^{2}}+\frac{q^{2}}{z^{4}}\right] y=0
$$

which shows the correspondence with the modified Bessel function. Now with the normalization that we have used in the representation (6.1) we shall need to know the values of $y^{\prime}(\xi) / y$ and $y^{\prime \prime}(\xi) / y$ at $\xi=\xi_{0}$ in order to implement the boundary conditions. The second ratio is given directly from (6.4). The first ratio is equal to $z p(z)$, where $p(z)=y^{\prime}(z) / y$ and $p$ satisfies the corresponding Ricatti equation

$$
\frac{\mathrm{d} p}{\mathrm{~d} z}+p^{2}+\frac{1}{z} p-\left(1+\frac{\alpha}{z^{2}}+\frac{q^{2}}{z^{4}}\right)=0
$$

for this ratio. An asymptotic analysis of the Ricatti equation as $z \rightarrow \infty$ gives the following behaviour:

where

$$
p \sim \sum_{n=0}^{\infty} A_{n} z^{-n} \quad \text { as } \quad z \rightarrow+\infty
$$

and

$$
\begin{aligned}
& A_{0}=-1, \quad A_{1}=-\frac{1}{2}, \quad A_{2}=-\frac{1}{2}\left(\alpha-\frac{1}{4}\right), \quad A_{3}=-A_{2}, \\
& A_{4}=-\frac{1}{2}\left[\frac{3}{2}\left(\alpha-\frac{1}{4}\right)-\frac{1}{4}\left(\alpha-\frac{1}{4}\right)^{2}+q^{2}\right] \\
& A_{n}=\frac{1}{2}\left\{(2-n) A_{n-1}+2 \sum_{k=1}^{\frac{1}{2}(n-1)} A_{k} A_{n-k}\right\} \quad(n \text { odd }), \\
& A_{n}=\frac{1}{2}\left\{(2-n) A_{n-1}+2 \sum_{k=1}^{\frac{1}{2}(n-2)} A_{k} A_{n-k}+A_{\frac{1}{2}}^{2}\right\} \quad(n \text { even }) .
\end{aligned}
$$

There are actually two asymptotic solutions. The other solution has leading behaviour +1 rather than -1 . We choose the minus sign corresponding to the exponentially decaying solution in $y$. To compute the value of $p\left(z_{0}\right)$ we integrate backwards from some sufficiently large value $z_{\infty}$ using the starting value given by the asymptotics. A variable-step Taylor-series routine was written especially to perform this integration. The number of terms in the asymptotic expansion and the value of $z_{\infty}$ was chosen according to the usual optimal asymptotic approximation rule (Bender \& Orszag 1978). This gave a very good estimate for $p\left(z_{\infty}\right)$. Although the desired solution is not asymptotically stable for increasing $z$, it is the stable solution for decreasing $z$, and one would expect to find good results at $z_{0}$ even for a poor starting value provided that $z_{\infty} \gg z_{0}$. The values computed by the routine were compared with tables of Bessel 


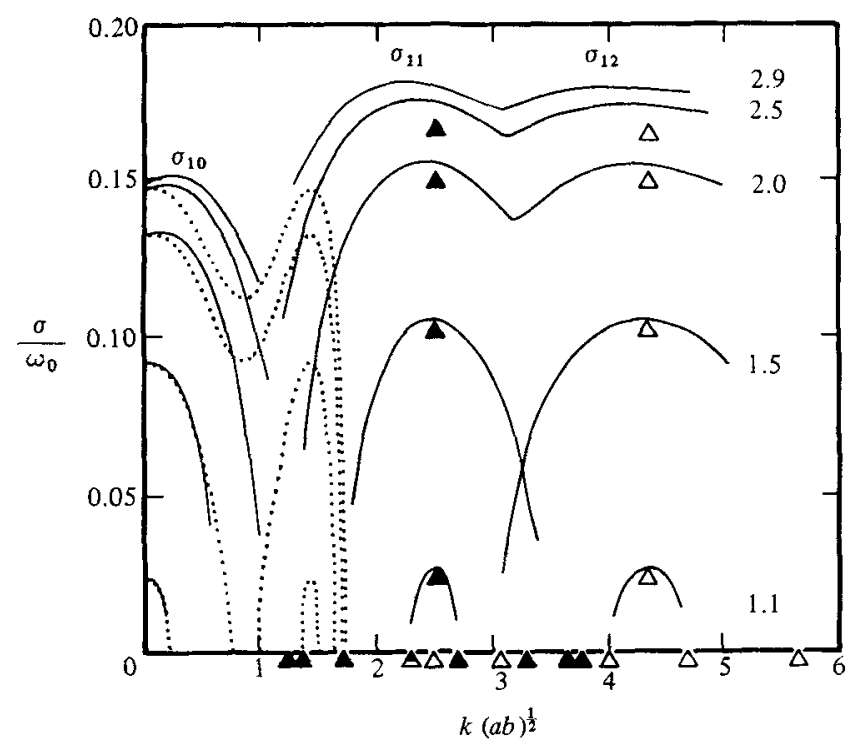

Figure 3. Values of $\sigma_{10}, \sigma_{11}$, and $\sigma_{12}$ versus axial wavenumber $k$ for $\theta=1.1,1.5,2.0,2.5$ and 2.9. Solid (open) triangles give predictions of Tsai \& Widnall (1976) for magnitude and width of instability about $k(a b)^{\frac{1}{2}}=2.5(4.35)$. Vertex of each triangle is plotting point. Dotted lines give prediction of $\sigma_{10}$ growth rate based on the Biot-Savart cutoff theory.

and Mathieu functions (Abramowitz \& Stegun 1972; Wiltse \& King 1958). A further self-consistency check was made by computing $p^{\prime}$ numerically using the values of $p$ output from the routine and checking these against the exact value. Excellent agreement was obtained over a wide range of parameter values.

The equations are non-dimensionalized in the following manner. 'The timescale is defined by the value of the vorticity in the undisturbed vortex, and the lengthscale by the geometric mean of the semimajor and semiminor axes. Then we have $\hat{\sigma}=\sigma / \omega_{0}$ and $\hat{k}=k(a b)^{\frac{1}{2}}$. The value of $\xi_{0}$ is given by the formula $\xi_{0}=\frac{1}{2} \log [(\theta+1) /(\theta-1)]$, and $c^{2} / a b$ is equal to $\left(\theta^{2}-1\right) / \theta$. The quantities $c$ and $k$ appear in the equations only as a product squared, which gives $q=\frac{1}{4}(c k)^{2}=\hat{k}^{2}\left(\theta^{2}-1\right) / 4 \theta$.

\section{Results and discussion}

We now describe the results of the computation of the growth rate of the zero-crossing eigenvalues as a function of the axial wavenumber and the axis ratio. The eigenvalues $\sigma$ are pure real in the cases to be described. Figure 3 shows the values of the growth rate for values of $\theta$ in the range $1.1-2.9 \approx \theta_{\mathrm{cr}}$. Also shown are the values of the growth rate and the region of instability for each mode as predicted by the perturbation analysis of Tsai \& Widnall (1976). It is seen that the numerical results match the perturbation results extremely well for small values of the strain. The effect of larger strain is weak relative to the perturbation results but several interesting features are observed. Note that the value of the axial wavenumber of maximum instability decreases slightly with increasing strain for the modes with one and two internal radial nodes starting at $\hat{k}=2.5$ and 4.35 respectively. These modes are labelled $\sigma_{11}$ and $\sigma_{12}$, where the first subscript denotes the angular dependence and the second the number of radial nodes for the nearly circular case. These modes meet 


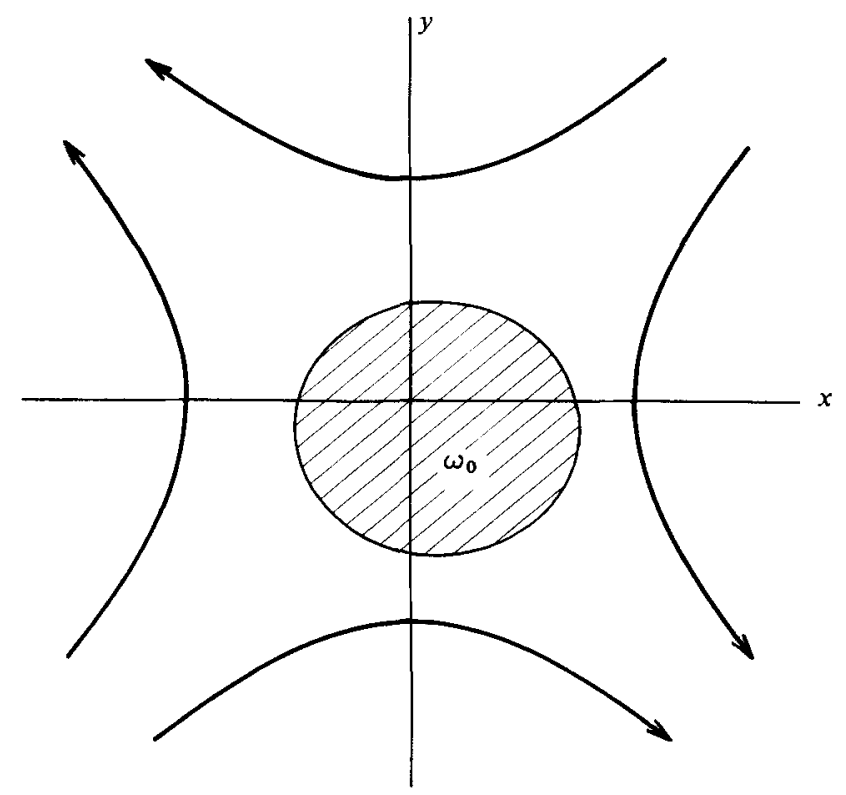

Figure 4. Pure two-dimensional $\sigma_{10}$ mode for $\theta=1.1$.

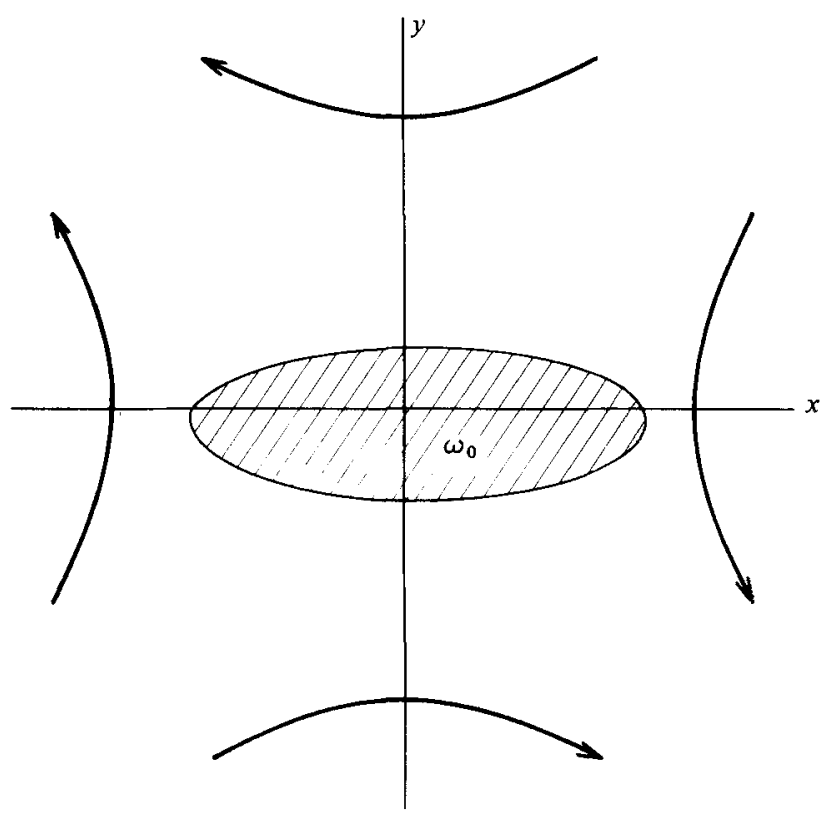

Figure 5. Pure two-dimensional $\sigma_{10}$ mode for $\theta=2.9$.

at $\theta \approx 1.4$ as the strain is increased. The growth-rate curves then cross as seen in the figure for $\theta=1.5$ and apparently also cross at larger $\theta$, although these details were not resolved. The $\sigma_{11}$ maximum is always the largest. The $\sigma_{10}$ and the $\sigma_{11}$ growth-rate curves also meet at about the value of the maximum strain. Notice in particular that the total range of unstable wavenumbers is very large for large strain and that the magnitudes of the growth rates for a significant portion of the wavenumber space 


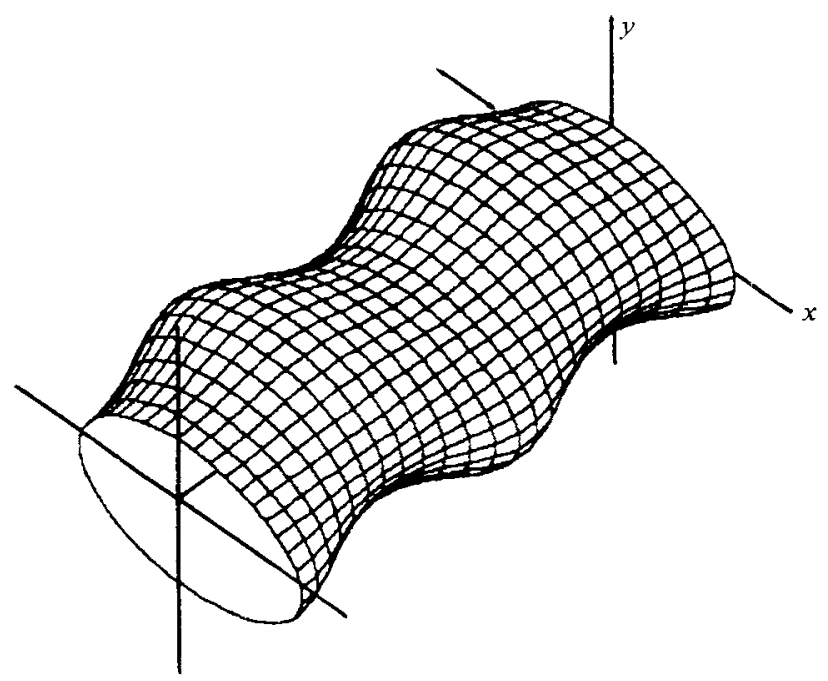

FigUre 6 . Short axial wavelength $\sigma_{11}$ mode for $\hat{k}=2.4$ amd $\theta=1.5$.

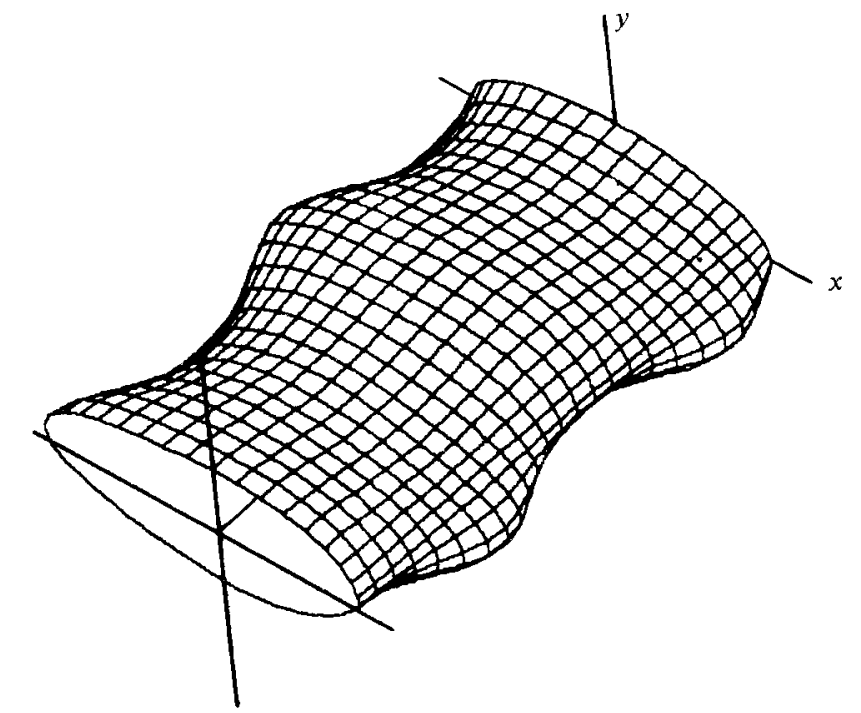

Figure 7. Short axial wavelength $\sigma_{11}$ mode for $\hat{k}=2.2$ and $\theta=2.5$.

are quite comparable. This suggests the possibility that observed instabilities in real flows might be more sensitive to the properties of external disturbances than to the structure of the vortex.

The long-wavelength $\sigma_{10}$ mode as predicted by the Biot-Savart cutoff theory is also shown in figure 3 . The dotted lines indicate the growth rate

$$
\hat{\sigma}=\left\{\left(\frac{e}{\omega_{0}}\right)^{2}-\left(\frac{1}{4} \hat{k}^{2}\left[\ln \frac{2}{\hat{k}}-\gamma+0.25\right]\right)^{2}\right\}^{\frac{1}{2}}
$$

where we have replaced the lengthscale for the radius of the vortex in the cutoff theory by the lengthscale $(a b)^{\frac{1}{2}}$. The value of $\gamma \approx \mathbf{0 . 5 7 7 2}$ is Euler's constant. Note the 


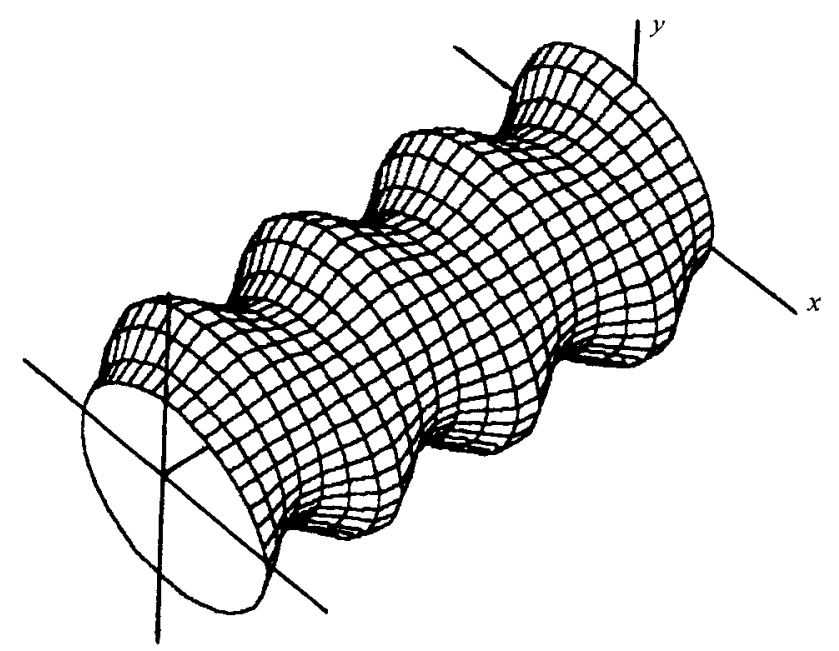

Figure 8. Short axial wavelength $\sigma_{12}$ mode for $\hat{k}=4.35$ and $\theta=1.1$.

excellent agreement for small values of the strain at small wavenumber. The growth rate decreases with increasing $k$ owing to the stabilizing effect of the vortex self-induction. For finite strain, however, the most-unstable long-wavelength mode is no longer purely two-dimensional but has a maximum at a finite value of the axial wavelength. Nevertheless, this effect is quite small and the general characteristics predicted by the cutoff theory are retained for the long-wavelength axial mode even for finite strain. This is a satisfying result relative to previous asymptotic studies, since it indicates that the cutoff theory, which assumes circular vortices moving in external strain, gives the correct long-wavelength stability behaviour for steady vortex configurations in which the size of the vortices relative to their separations need not be very small and the vortices may take on an elliptical cross-section. It is particularly noteworthy that the spurious instability predicted by the cutoff theory is not qualitatively different from the real instability of the $\sigma_{11}$ mode, which explains why some incorrect theories of vortex-ring instability gave apparently correct results.

Of further interest is the character of the eigenfunctions themselves. For this purpose we show in figures 4-7 the surface of the deformed vortex where the surface is given by (5.3) and $F$ is given from the eigenfunction computation. In the case of the pure two-dimensional $m=1$ mode we give the equation explicitly as

$$
\xi=\xi_{0}+\epsilon \frac{\cos \left(\eta \pm \operatorname{Tan}^{-1} \theta\right)}{h^{2}\left(\xi_{0}, \eta\right)}
$$

where the signs correspond to the plus and minus eigenvalues respectively and $\epsilon$ is a sufficiently small arbitrary amplitude. It is clear that the unstable mode represents the translation of the ellipse outward along one of the two outgoing principal axes of strain as mentioned by Moore \& Saffman (1971). Figures 4 and 5 show the disturbed vortex in cases of weak and strong external strain. For the short-axial-wavelength modes, we plot a three-dimensional view of the surface of the vortex given by the normal-mode calculation. Figures 6 and 7 give a plot of the deformed vortex for the $\sigma_{11}$ instability for representative values of $k$ and $\theta$. Figures 8 and 9 give similar views for the $\sigma_{12}$ instability. Note in all cases the definite bulging and orientation in the direction of the outgoing strain. The spanwise length shown is five times the vortex cross-sectional lengthscale. 


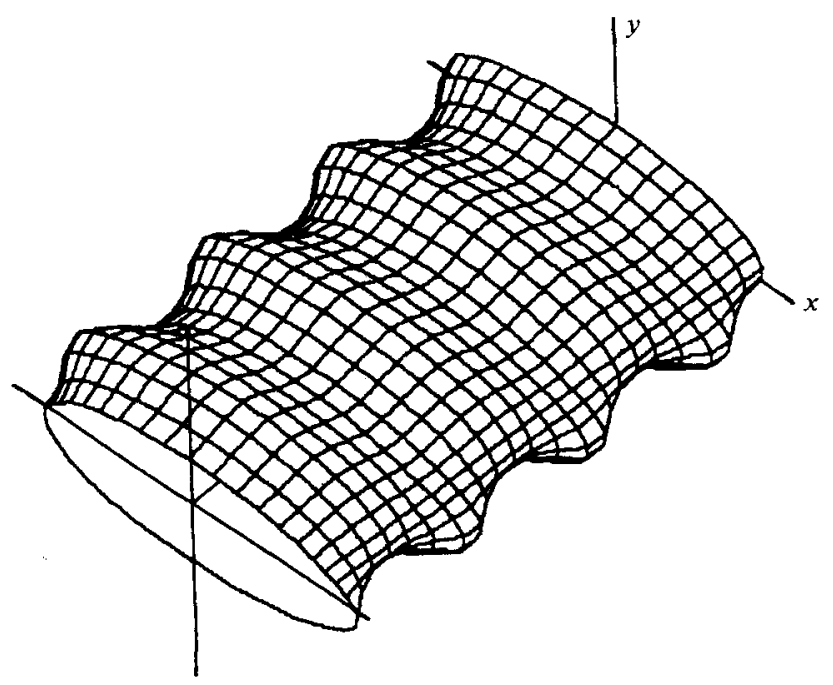

Figure 9. Short axial wavelength $\sigma_{12}$ mode for $\hat{k}=4.3$ and $\theta=2.9$.

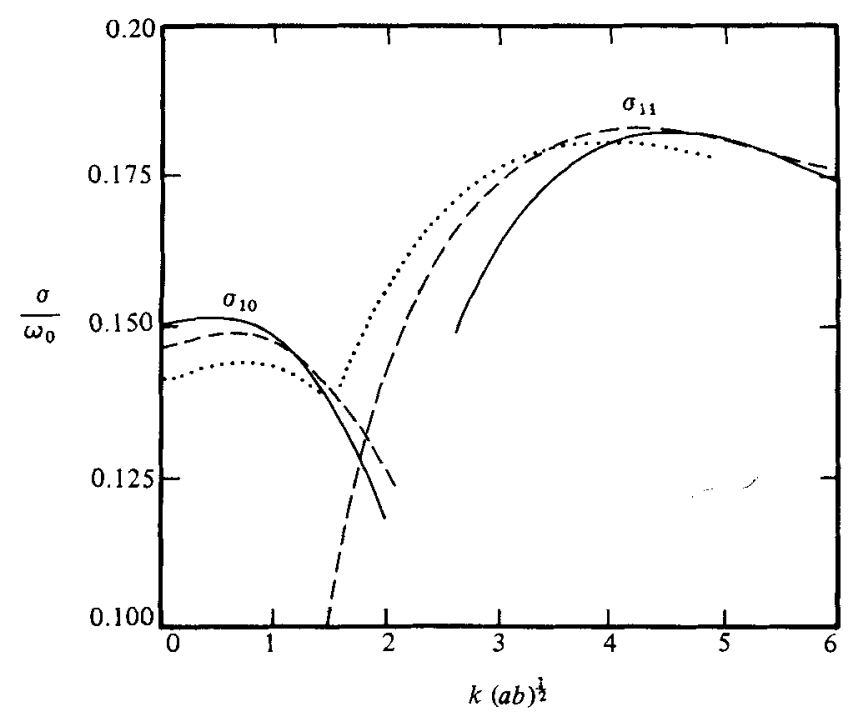

Figure 10. Values of $\sigma_{10}$ and $\sigma_{11}$ for $\theta \geqslant \theta_{\text {cr }}$ versus axial wavenumber $k$ for $\theta=2.9$ (solid), 3.5 (dashed) and 4.0 (dotted).

We look now at the steady state for $\theta \geqslant 2.9$ and show the variation of the parametric instability with $\theta$. The flow is not unstable to two-dimensional structural instabilities of $\pi$-periodic type, but it is of interest to see how the growth rate for the three-dimensional disturbances varies as $\theta$ increases, at least until the threedimensional and the two-dimensional growth rates are comparable. Figure 10 shows for the three values of $\theta$ the dependence of the $\sigma_{10}$ and $\sigma_{11}$ modes on $k$. With regard to the dependence on $\theta$, the most-unstable long-wavelength mode is seen to decrease in magnitude while still maintaining its three-dimensional character, while the short-wavelength mode is seen to increase initially as $\theta$ increases above $\theta_{\mathrm{cr}}$ and then decreases as $\theta$ is increased further. For $\theta=4.0$ we can see from (3.1) with $m=2$ that 
the growth rate for the two-dimensional structural instability is $\sigma=0.1776$ and is thus of the same order as the three-dimensional instability.

In summary we have shown that the effect of finite strain on the three-dimensional $2 \pi$-periodic modes studied is to increase the growth rates above the values predicted by the perturbation theory and make them practically independent of wavenumber. The effect of large deformation in the steady solution thus does not ameliorate the three-dimensional instability but removes the tuning or tendency to select a particular wavenumber. For $\theta$ less than about 4.0 the three-dimensional instabilities have larger growth rates than the two-dimensional instabilities. It is also seen that the characteristies of the long-wavelength mode are not significantly affected by finite strain. This further justifies the use of the Biot-Savart formulation as a useful tool to analyse three-dimensional long-wavelength instabilities of rectilinear vortex arrays.

This work was supported by NASA Lewis Research Center (NAG 3-179) and the Office of Naval Research.

\section{REFERENCES}

Abramowitz, M. \& Stegun, I. A. 1972 Handbook of Mathematical Functions. Natl Bur. Stand./Dover.

Batchelor, G. K. 1967 An Introduction to Fluid Dynamics. Cambridge University Press.

Bender, C. M. \& ORszag, S. A. 1978 Advanced Mathematical Methods for Scientists and Engineers. McGraw-Hill.

Blanch, G. 1966 Numerical aspects of Mathieu eigenvalues. Rend. Circ. Mat. Palermo (2) 15, 51-97.

Boy D, J. P. 1978 Spectral and pseudospectral methods for eigenvalue and nonseparable boundary value problems. Mon. Weather Rev. 106, 1192-1203.

CLEMм, D. S. 1969 Algorithm 352: Characteristic values and associated solutions of Mathieu's differential equation. Comm. ACM 12, 399-407.

Garbow, B. S., Boyle, J. M., Dongarra, J.J. \& Moler, C. B. 1977 Matrix Eigensystem Routines - EISPACK Guide Extension. Lecture Notes in Computer Science vol. 51. Springer.

Kelvin, Lord 1880 Vibrations of a columnar vortex. Phil. Mag. 10, 155-168.

KIDA, S. 1981 Motion of an elliptic vortex in a uniform shear flow. J. Phys. Soc. Japan 50, $3517-3520$.

Moore, D. W. \& Saffman, P. G. 1971 Structure of a line vortex in an imposed strain. In Aircraft Wake Turbulence (ed. Olsen, Goldburg \& Rogers), pp. 339-354. Plenum.

Moore, D. W. \& SAFFman, P. G. 1975 The instability of a straight vortex filament in a strain field. Proc. R. Soc. Lond. A 346, 413-425.

Nev, J. 1983 The dynamics of a columnar vortex in an imposed strain. Math. Sci. Res. Inst. Rep. 022-83, Berkeley, Calif.

Pierrenumbert, R. T. 1980 The structure and stability of large vortices in an inviscid flow. M.I.T. Fluid Dym. Lab. Rep. 80-1.

Pierrehumbert, R. T. \& Widnald, S. E. 1982 The two- and three-dimensional instabilities of a spatially periodic shear layer. J. Fluid Mech. 114, 59-82.

Robinson, A. C. \& Safrman, P. G. 1982 Three-dimensional stability of vortex arrays. J. Fluid Mech. 125, 411-427.

SaffMan, P. G. 1978 The number of waves on unstable vortex rings. J. Fluid Mech 84, 625-639.

SALF, A. H. J. 1970 Remark on algorithm 352 [S22]; Characteristic values and associated solutions of Mathieu's differential equation. Comm. ACM 13, 750.

TsaI, C-Y. \& Widnald, S. E. 1976 The stability of short waves on a straight vortex filament in a weak externally imposed strain field. J. Fluid Mech. 73, 721-733.

WARD, R. C. 1981 Balancing the generalized eigenvalue problem. SIAM J. Sci. Stat. Comp. 2 , 141-152. 
Widnall, S. E., Bliss, D. B. \& TSAI, C-Y. 1974 The instability of short waves on a vortex ring. J. Fluid Mech. 66, 35-47.

Widnall, S. E. \& Tsar, C-Y. 1977 The instability of the thin vortex ring of constant vorticity. Phil. Trans. R. Soc. Lond. A 287, 273-305.

Wiltse, J. C. \& King, M. J. 1958 Values of the Mathieu functions. The Johns Hopkins Univ. Rad. Lab. Tech. Rep. AF-53, Baltimore, Md. 